\title{
Forming Uniform HD Layers in Shells Using Infrared Radiation
}

\author{
D. N. Bittner \\ G. W. Collins \\ E. Monsler \\ S. Letts
}

This paper was prepared for submittal to the 40th Annual Meeting of the Division of Plasma Physics

New Orleans, LA

November 16-20, 1998

October 12, 1998

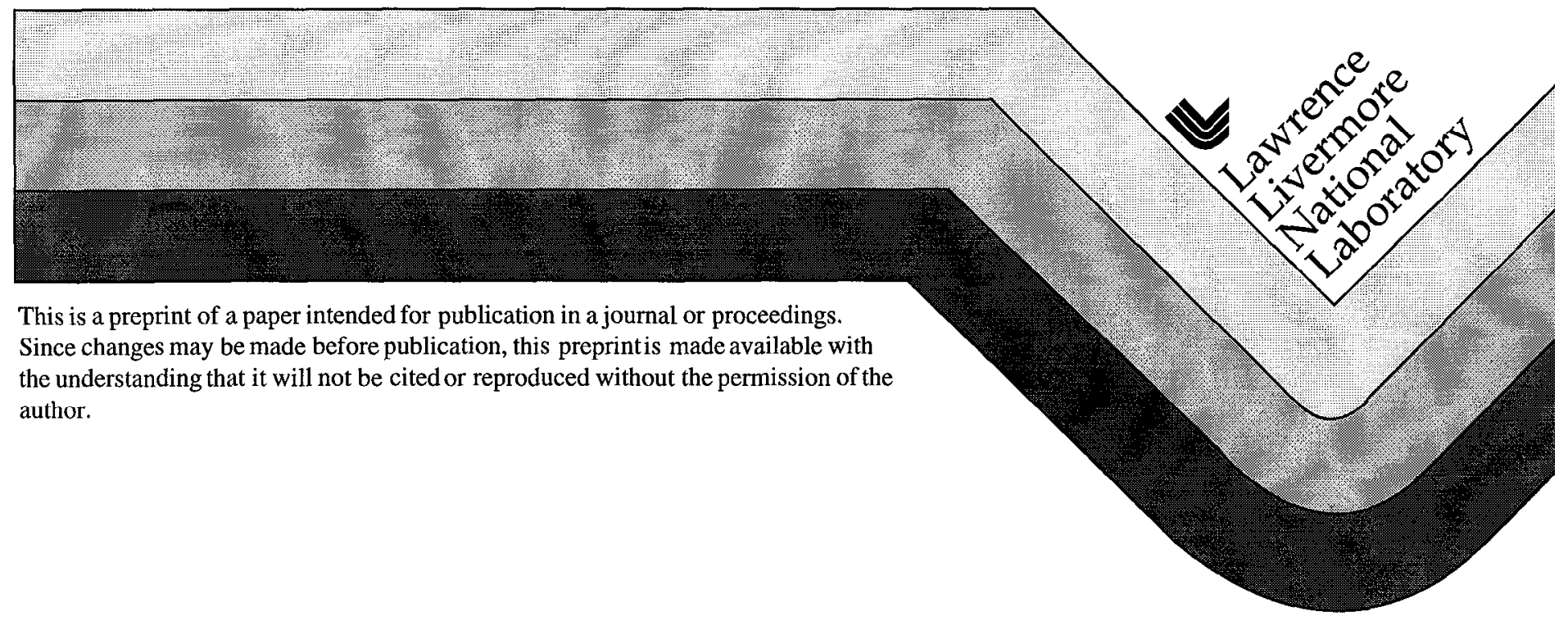




\section{DISCLAIMER}

This document was prepared as an account of work sponsored by an agency of the United States Government. Neither the United States Government nor the University of California nor any of their employees, makes any warranty, express or implied, or assumes any legal liability or responsibility for the accuracy, completeness, or usefulness of any information, apparatus, product, or process disclosed, or represents that its use would not infringe privately owned rights. Reference herein to any specific commercial product, process, or service by trade name, trademark, manufacturer, or otherwise, does not necessarily constitute or imply its endorsement, recommendation, or favoring by the United States Government or the University of California. The views and opinions of authors expressed herein do not necessarily state or reflect those of the United States Government or the University of California, and shall not be used for advertising or product endorsement purposes. 


\title{
FORMING UNIFORM HD LAYERS IN SHELLS USING INFRARED RADIATION
}

\author{
D.N. Bittner ${ }^{1}$, G.W. Collins ${ }^{2}$, E. Monsler ${ }^{1}$, S. Letts ${ }^{2}$ \\ ${ }^{1}$ Schafer Corporation \\ 303 Lindbergh Ave. \\ Livermore, CA 94550 \\ 2 Lawrence Livermore National Laboratory \\ P.O. Box 808 \\ Livermore, CA 94551
}

\begin{abstract}
Generating a volumetric heat source in solid deuterium hydride, HD, allows the formation of a spherical crystalline shell of HD inside a transparent plastic shell. Pumping the infra-red (IR) collisionally induced vibration-rotation band of solid HD contained inside a transparent plastic shell generates the volumetric heat source in the HD lattice. HD layers $150-250 \mu \mathrm{m}$ thick formed near the triple point have a surface roughness rms between $1-3 \mu \mathrm{m}$ and become rougher with decreasing temperature. Solid growth dynamics have a significant impact on the quality of the resultant layer.
\end{abstract}

\section{INTRODUCTION}

Smooth and uniform 50 to $300 \mu \mathrm{m}$ thick deuteriumtritium (DT) layers on the interior of 1 to $3 \mathrm{~mm}$ diameter spherical capsules are required for ignitable inertial confinement fusion (ICF) targets for the National Ignition Facility (NIF). ${ }^{1}$ One way to form these layers is to initially freeze DT to an anisotropic multicrystalline solid inside a capsule and then allow the tritium decay volumetric heat generation, $\mathrm{Q}_{\mathrm{DT}}=0.05 \mathrm{~W} / \mathrm{cc}$, to symmetrize the layer. The volumetric heating causes the thicker regions of the solid to have a higher temperature and thus a higher vapor pressure. This results in a redistribution of the solid until the inner DT surface is isothermal. The time constant for this redistribution (when no ${ }^{3} \mathrm{He}$ is present) is $\tau_{\mathrm{o}}=\mathrm{H}_{s} \rho / \mathrm{Q}_{\mathrm{DT}}=23$ minutes, where $\mathrm{H}_{s}$ is the heat of sublimation ( $\mathrm{J} / \mathrm{mole}$ ), and $\rho$ is the density (moles/cc). ${ }^{2}$ If bulk heating were the only factor controlling the layer profile, DT would form a uniform spherical layer inside an isothermal spherical capsule. However, crystal surface stiffness contributes to the final layer shape. The surface smoothes until the thermal energy associated with material redistribution is comparable to the surface energy gained in having to form a higher energy viscinal surface. ${ }^{3}$ The surface structure of a multicrystalline DT film is a function of the DT bulk heating ratc and the distribution of crystallite sizes and orientations which are determined by the initial nucleation and growth. ${ }^{4}$

Several implosion hydrodynamics issues of cryogenic targets can be investigated with deuterium hydride (HD) or deuterium $\left(\mathrm{D}_{2}\right)$ without the hazards of handling high pressure tritium. To generate bulk heat for mass redistribution without tritium radiation, we pump the collisionally induced infra-red (IR) vibration-rotation band. We regulate the solid volumetric heating rate, $Q_{I R}$, and thus the redistribution rate, $1 / \tau_{I R}$, and surface roughness by controlling the incident IR intensity. We have measured laser power limited $1 / \tau_{\mathrm{IR}}$ (and thus $\mathrm{Q}_{\mathbb{R}}$ ) in HD up to ten times higher than the DT value. The maximum $Q_{\mathbb{R}}$ for hydrogen isotopes is limited by the vibrational relaxation time to $\mathrm{Q}_{\mathrm{IR}} \sim 1000 \mathrm{Q}_{\mathrm{DT}}{ }^{\circ}$. These large $Q_{I R}$ values enable us to control the hydrogen layer profile as well as explore the redistribution process of laser heated solids.

\section{EXPERIMENTAL ASPECTS}

\section{A. Experimental Overview}

We have previously measured redistribution time constants, $\tau_{\mathrm{IR}}$, from IR illumination of both solid $\mathrm{HD}$ and $D_{2}$ at several different wavelengths versus incident IR flux to determine $Q_{\mathrm{IR}}{ }^{6.7}$ These redistribution experiments showed that the illumination of a solid sample with IR radiation resulted in the redistribution of the solid analogous to that seen with tritium heat generation in DT. In a second series of experiments using a similar experimental design but with a larger sapphire cell, the surface roughness of a layer formed by illuminating the solid with IR was investigated. The illumination pattern of the IR was found to have significant influence on the layer shape. The resultant phase shifting interferometric analysis revealed that surface perturbations with spatial extent greater than the layer thickness have amplitudes which decrease with increasing volumetric heating. The minimum roughness achieved at a given volumetric 
heating rate was found to be dependent on the nucleation and subsequent crystal growth during freezing. Both of these experiments were effectively one dimensional. A laser beam made one pass coaxially along the inner bore of the cell and measurements were made by looking through the side of the container or coaxially along the bore of the cell. In the present experiments we generate a $4 \pi$ illumination of $H D$ inside a $1 \mathrm{~mm}$ O.D. spherical capsule and observe the evolution of a uniform solid layer as a function of time and IR intensity.

\section{B. Experimental Design and Layout}

In designing these experiments several issues needed to be addressed. The first issue was the choice of capsule material, $C D$ plasma polymer. The goal is to heat the $\mathrm{HD}$ $\left(D_{2}\right.$ or DT) and not the plastic shell. Therefore, it is important to minimize the amount of IR absorption (heating) by the capsule. Standard plasma polymer, $\mathrm{CH}_{1,3}$, used in current NOVA experiments has several absorptions overlapping the absorption band for both $D_{2}$ and HD. Thus it would be difficult to redistribute HD or $\mathrm{D}_{2}$ in shells made of this material. These absorption bands are primarily $\mathrm{CH}$ and $\mathrm{OH}$ stretch modes. There is also a broad band loss of transmission that extends over the entire spectral range of measurement $\left(400-5125 \mathrm{~cm}^{-1}\right)$ which may be due to IR scattering. Therefore we explored alternatives and found that a $C D$ plasma polymer made with deuterated $\mathrm{p}$-Xylene monomer and $\mathrm{D}_{2}$ flow gas does not have corresponding strong $\mathrm{CH}$ absorption lines and also has good broadband transmission. Further, while the $\mathrm{OH}$ absorption band increased with time in air, it is controlled by keeping the shell in a dry box. This was the shell material used for these experiments (Fig. 1).

A second issue was the technique for generating a uniform IR illumination. The surface roughness experiments showed that diffuse illumination was necessary to reduce laser induced coherence effects. Calculations showed that illumination uniformity to better than $99 \%$ could be achieved ${ }^{8}$ by using an integrating sphere. The integrating sphere used for these experiments was a vacuum tight enclosure made from aluminum with four window ports and an Infragold coating $^{9}$ on the inner surface. The Infragold surface is greater than $96 \%$ reflecting at wavelengths of interest.

While a systematic study of surface roughness as a function of $\mathrm{Q}_{\mathrm{IR}}$ was not performed, the smoothest surfaces were generated at $Q_{\mathbb{R}} \approx 200 \mathrm{~mW} / \mathrm{cc}$. Using this value one can estimate the IR power requirements for the integrating sphere. The volumetric heating rate is related to the IR intensity by $Q_{\mathbb{R}}=\alpha I$, where $\alpha$ is the absorption coefficient $(\alpha(H D) \approx 8 / \mathrm{cm})$ and I is the intensity. The requisite volumetric heating rate is produced by injecting sufficient IR into the integrating sphere to produce the required intensity $I_{0}=Q_{I R} / \alpha$. For weakly absorbing layers, $I \approx I_{0}$, the incident intensity. The capsule is small compared to that of the integrating sphere resulting in most of the power losses occurring at the integrating sphere wall and through the viewports. Noting that the injected power only needs to be sufficient to compensate for integrating sphere losses (about $4 \%$ ), this results in a linear relationship between the laser power and the incident intensity, $P_{I S}=(0.04) I_{0} A_{I S}$, where $P_{I S}$ and $A_{I S}$ are the injected IR power and surface area of the integrating sphere, respectively. Thus, an intensity of $\mathrm{I}_{\mathrm{o}}=$ $25 \mathrm{~mW} / \mathrm{cm}^{2}$ incident on the $1 \mathrm{~mm}$ O.D. capsule in the center of a $25 \mathrm{~mm}$ I.D. integrating sphere requires $21 \mathrm{~mW}$ of IR.

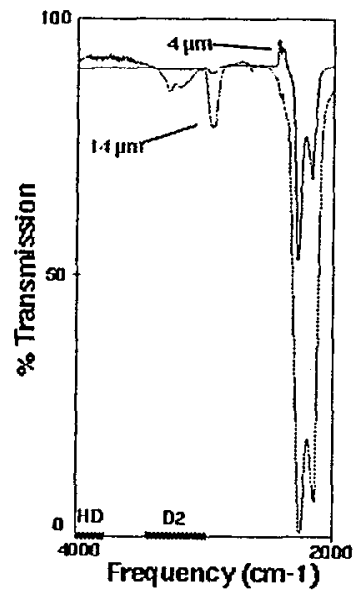

Fig. 1 IR transmission spectra of $4 \mu \mathrm{m}$ and a $14 \mu \mathrm{m}$ thick $\mathrm{CD}$ plasma polymer films. The overlapping absorption band ranges for $\mathrm{HD}$ and $\mathrm{D}_{2}$ are indicated along the bottom of the plot.

Finally, asymmetries in the solid layer due to perturbations in the thermal environment caused by the fill tube needed to be minimized. The fill tube essentially acts as a cooling fin on the shell. The thermal conductance through the fill tube is greater than that of the surrounding helium exchange gas. This causes the shell area around the fill tube to be cooler than the rest of the capsule resulting in a thicker layer forming in this region. Various means of attaching a fill tube to the capsule were investigated and modeled using the finite element code COSMOS. The final design involved overcoating a composite shell/fill tube mandrel with plasma polymer. The resulting capsule was nominally $1 \mathrm{~mm}$ O.D. with a $10 \mu \mathrm{m}$ thick wall seamlessly attached to a 1-2 mm long by $100 \mu \mathrm{m}$ O.D. plasma polymer fill tube extension. ${ }^{10}$ 


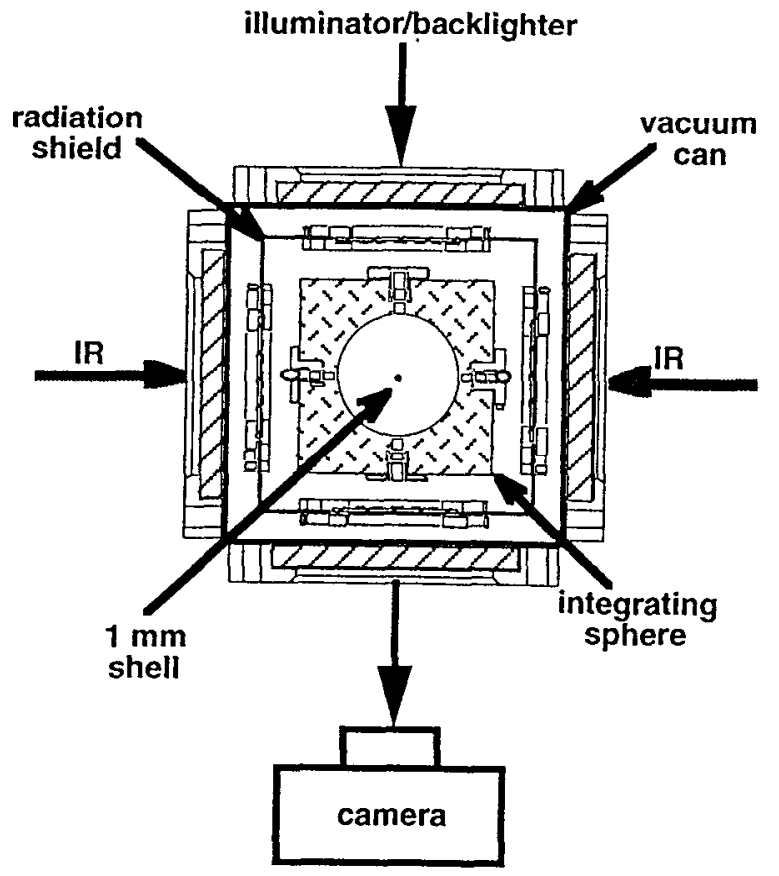

Fig. 2 Experimental layout indicating the viewing axis and the IR injection axis. The sapphire ball lenses on the integrating sphere ports spread the IR beam in an $\mathrm{f} / 1$ conc.

Figure 2 contains a sketch of the experimental layout. For these experiments a $\mathrm{CD}$ shell containing $\mathrm{HD}$ was placed in the center of a $25 \mathrm{~mm}$ I.D. integrating sphere filled with roughly 0.5 Torr of helium exchange gas. The fill line was composed of three sections: a $0.82 \mathrm{~mm}$ long $\mathrm{CD}$ tube attached to a $5 \mathrm{~mm}$ long glass tube which was in turn attached to a stainless steel tube. The stainless tube extended through the integrating sphere wall. To minimize IR absorption in the fill line, the glass tube was gold coated. The shell containing HD was illuminated through one pair of viewports by an IR laser beam and the layer monitored along a second orthogonal axis by backlighting the capsule through one port and using a Questar microscope on the opposite port for viewing. Images were acquired periodically during layer formation using a Photometrics 1317 by 1035 array CCD camera which is attached to the microscope. Real time monitoring of the layer was done with a Cohu camera mounted on the microscope eyepiece port. The integrating sphere was thermally and mechanically attached to the cold tip of a helium flow cryostat.

\section{Data Collection and Analysis Procedures}

To determine the bulk heating rate we measured the redistribution time constant, $\tau_{\mathrm{IR}}$. This was accomplished by filling the capsule with $H D$, rapidly cooling to around $15 \mathrm{~K}$ to freeze the HD, and then turning on the IR and collecting images of the evolving layer with time. Typically the HD thickness was small near the top of the shell but grew exponentially with time to form a uniform layer. The exponential time constant is related to the volumetric heating rate by $\tau_{\mathrm{IR}}=\mathrm{H}_{s} \rho / \mathrm{Q}_{\mathrm{IR}}$.

Roughness of the inner solid surface was determined by performing a Fast Fourier Transform, FFT, analysis of the brightband in the CCD camera images. The brightband can be understood by performing a raytrace ${ }^{11}$ through a $1 \mathrm{~mm}$ O.D. shell containing a uniform layer inside an integrating sphere with $4 \mathrm{~mm}$ O.D. ports. The HD filled capsule acts as a weak negative lens resulting in light near the edge of the shell being refracted out of the field of view. Rays internally reflected off the inner solid surface do not leave the field of view and make it through the shell and integrating sphere viewport to the collection optics. Fig. 3a shows a closeup view of a bundle of rays incident on the capsule. Fig. $3 \mathrm{~b}$ is an expanded view showing these rays exiting the integrating sphere through a viewport. Bundles of rays incident on the shell at the largest incident angles consistent with our optical arrangement were traced and found to produce the same brightband.
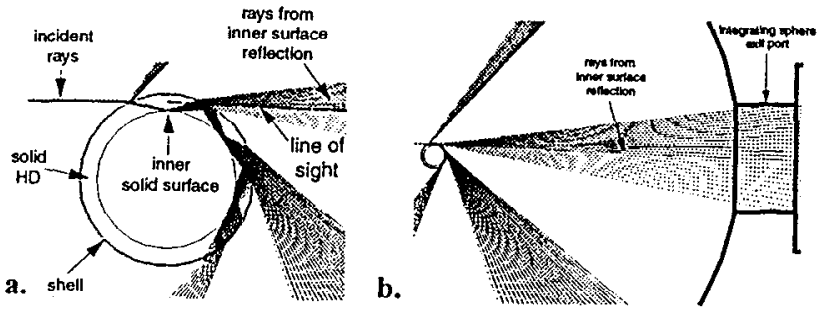

Fig. 3 Raytrace plot showing the reflection of light off the inner solid surface (a). Most of the light makes it out of the integrating sphere through the viewport (b).

Refraction also affects the determination of the layer thickness. Typically, the layer thickness is determined by acquiring an image with the $C C D$ camera, measuring the distance from the bright band to the edge of the shell in pixels, comparing that to the measured distance across the diameter of the shell, also in pixels, and finally scaling pixels to micrometers using the shell diameter previously determined by standard characterization techniques ${ }^{12}$. If the microscope is focused at the center plane of the shell, then by using this method one is assuming the rays from the bright band extrapolate in a straight line back to the center of the shell. Fig. 3a contains a dashed line extrapolating the rays to the center plane of the shell. The measured, or apparent, layer thickness determined from a camera image is less than the actual layer thickness by the difference between the dashed line and the inner solid 
surface. To estimate the actual layer thickness a simple model was generated to calculate both the apparent and the actual layer thicknesses inside a $10 \mu \mathrm{m}$ thick, $1 \mathrm{~mm}$ O.D. plastic shell. The results are presented in Fig. 4 and show that refractive effects have a significant impact on the measurement of thin layers, those less than $100 \mu \mathrm{m}$. A $100 \mu \mathrm{m}$ thick layer would appear to be $45 \mu \mathrm{m}$ thick on the image.

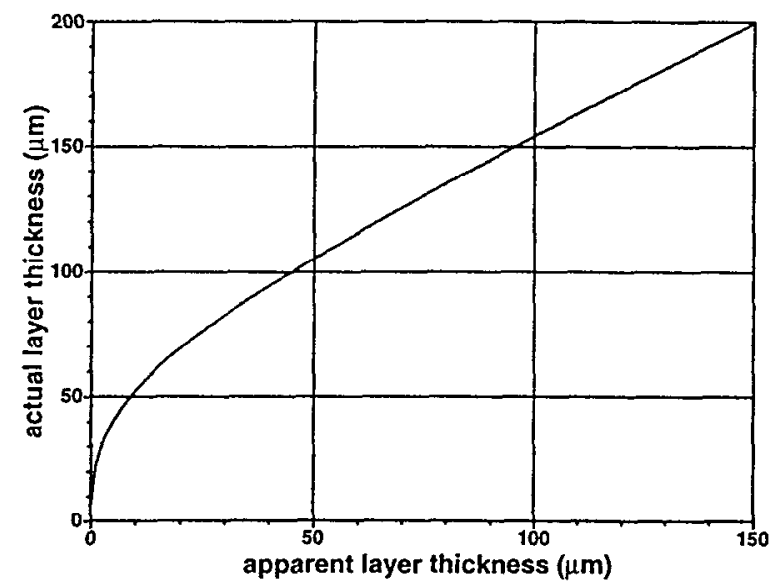

Fig. 4 Plot of apparent versus actual layer thickness inside a $10 \mu \mathrm{m}$ thick, $1 \mathrm{~mm}$ O.D. plastic shell.

Once the images have been shot and stored the objective of the experiment is to extract an estimate of the surface roughness from the stored data. Software has previously been developed which locates the surface of the solid in the image. ${ }^{3}$ The resultant one-dimensional estimate of the solid thickness variations are Fourier transformed to estimate the power spectrum of perturbations on the solid layer.

The software begins by taking an estimate of the center of the brightband in the image and drawing a ray from the center to the edge. At one pixel increments along the ray, the intensity is estimated by fitting a plane to the four neighboring pixel values and interpolating to get the intensity at the point on the ray. This is done for a series of rays offset by some given angular increment to generate an image of the target in polar coordinates. Each column in this image is then processed to locate the light to dark transition associated with the inner edge of the solid. The estimates of the locations of the edge of the brightband are averaged to give a new value of the center of the brightband, and the image is reprocessed until these estimates converge.

There is a large step in the intensity at the inner edge of the solid. Refraction at the inner edge of the solid creates intensity minima in the spherical geometry which are located using an algorithm to find the minima in each ray. The minima are sorted by depth for each ray to give an array of locations as a function of angle for each feature. These profiles can be Fourier transformed and the results used to calculate power spectra for the surfaces. In our case, since we do not locate the shell edge, we do not determine the offset of the inner surface from the capsule (Fourier mode 1), but fit Fourier modes 2 through 128.

\section{RESULTS AND DISCUSSION}

The layers grown by the rapid cooling technique typically result in rough multicrystalline surfaces. Fig. 5 shows the evolution of one of these rough layers in which the volumetric heating rate was about $250 \mathrm{~mW} / \mathrm{cc}$ (or $5 \mathrm{Q}_{\mathrm{DT}}$ ). One starts the layering process by performing a controlled liquid fill. Once sufficient liquid is inside the capsule (Fig. 5a) the temperature is quickly lowered to freeze the solid (Fig. 5b). The randomly grown solid is then illuminated with IR radiation and the redistribution of the solid is recorded with time. Fig. 5c shows the resultant layer.
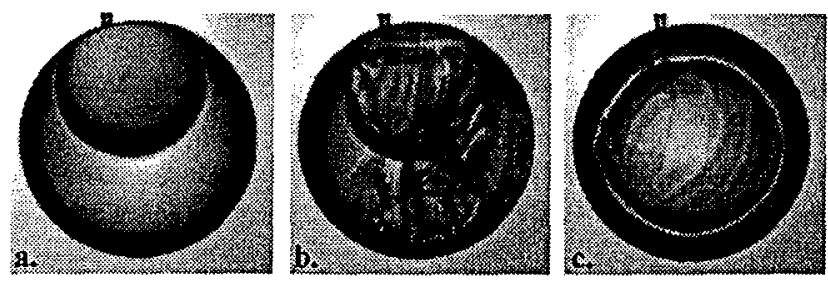

Fig. 5 To generate a layer from the solid, one fills the capsule with liquid at $16.6 \mathrm{~K}$ (a), lowers the temperature to $15.8 \mathrm{~K}$ to form solid (b), and then upon application of IR, the solid redistributes to form a layer (c). These layers typically have a rough surface, in this case even after 16 hours.

In order to improve the quality of the inner solid surface, a second technique was developed. Currently this technique is performed by manually entering the temperature into a temperature controller while viewing the layer evolution on a monitor. The following procedure was used to grow higher quality layers: freeze the $\mathrm{HD}$, inject IR into the integrating sphere, raise the temperature of the capsule until the solid just finishes melting, slowly lower the temperature in approximately $0.005 \mathrm{~K}$ steps until freezing onset occurs, at which point the temperature is no longer lowered. If solidification appeared to stop, then the temperature would be lowered in $0.005 \mathrm{~K}$ steps until a uniform layer had formed. Images of the evolving layer were reconded with time. Figure 6 shows an approximately $200 \mu \mathrm{m}$ thick layer grown by this technique. Figure 7 is the power spectrum for the solid layer in Fig. 6d. The resultant rms for this layer is 
$2.24 \mu \mathrm{m}$. This is slightly different from the slow growth technique used for DT layers. ${ }^{13}$ Since DT is a mixture, there is not a triple point as in a single component system $\left(D_{2}\right)$, but a three phase region. The slow growth technique cools the DT slowly through this region.
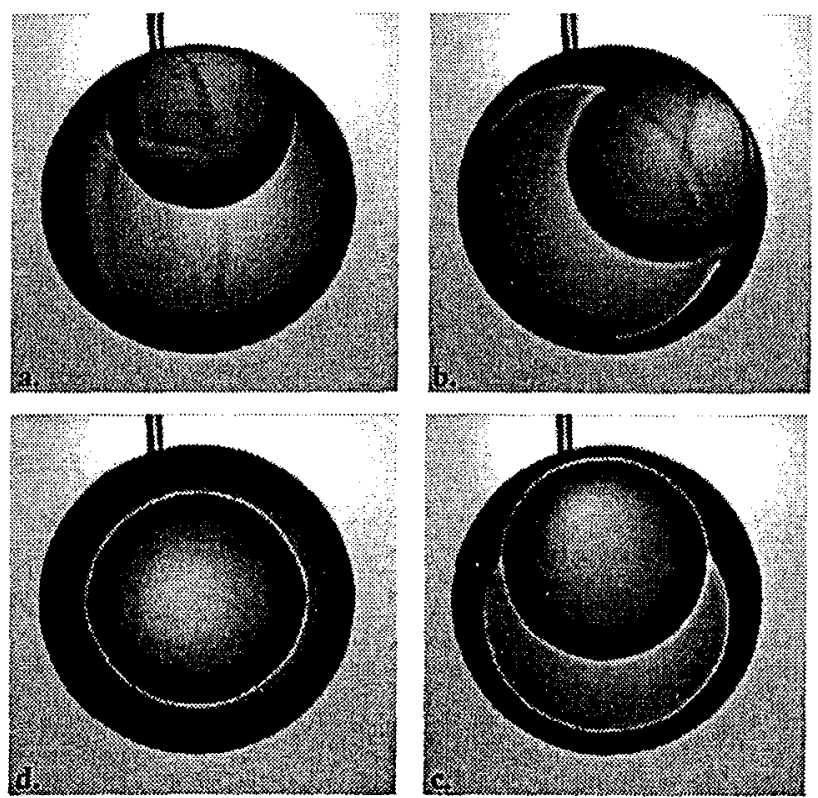

Fig. 6 In an improved layering technique IR is continually applied to the capsule. The capsule is then warmed to the liquid phase $(16.6 \mathrm{~K})$ and slowly cooled until solid starts forming (a. $\mathrm{t}=0 \mathrm{~min}$.). The temperature is then held constant during the solidification process (b. $t=19$ min., c. $t=32$ min., d. $t=62 \mathrm{~min}$.).

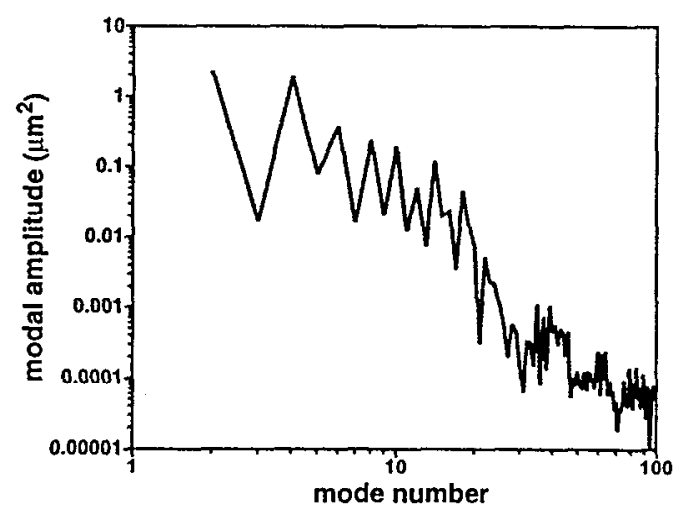

Fig. 7 Power spectrum for the layer shown in Fig. 6d. The noise level for this image can be seen by the flattening of the power spectrum at mode numbers above 50 . The rms value of this layer is $2.34 \mu \mathrm{m}$.

Laser power and temperature stability affect the growth of the solid. Higher quality layers were formed when the temperature was controlled to at least $\pm 5 \mathrm{mK}$ under stable laser power. The impact of the laser is twofold. In addition to generating a given volumetric heating rate, the absorbed energy also raises the temperature of the solid. Variations in laser power cause both volumetric heating rate and temperature variations. Large temperature fluctuations cause the HD to melt and freeze too rapidly resulting in rough layers. When both systems were stable, it typically took about one hour for layer completion.

As can be seen in Fig. $5 \mathrm{c}$ and $6 \mathrm{~d}$, there are features in the layers which are similar in both these images. One is a "bump" (or flat spot) near the fill tube. The fill tube has a higher thermal conductivity than the surrounding helium exchange gas producing a cold spot at the fill tube/shell junction and resulting in a thickening or "bump" in the layer. The only way to remove this feature is to eliminate the fill tube. The second pair of features can be seen at the shell equator. These features are due to the IR injection method. The IR is injected along an axis through the equator of the shell resulting in more intense illumination there than from the rest of the integrating sphere. This causes locally warmer areas resulting in thin spots in the layer. These features can be removed by alternative injection techniques such as shaping the beam, inserting a beam block in front of the shell, or, more easily, by initially directing the beam to miss the shell instead of passing through it.

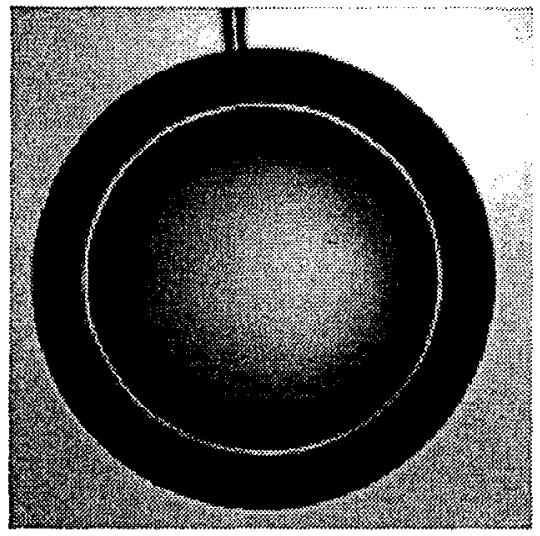

Fig. 8 Layer form by direct injection of the IR laser beam after removal of the sapphire ball lenses. Thinning of the layer at the capsule equator is no longer evident.

Figure 8 shows a $180 \mu \mathrm{m}$ layer produce by directly injecting the beam into the integrating sphere. For this arrangement the sapphire balls were removed and one beam was injected off axis at an angle of $11^{\circ}$ through the intcgrating sphcre window. This permitted the approximately $2.5 \mathrm{~mm}$ diameter beam to pass through the window, miss the shell, and hit the integrating sphere wall. One can still detect a thickening of the layer near the fill tube but the thin spots at the cquator seen in the 
previous arrangement are gone. Layers grown using IR illumination are comparable to DT layers grown by the slow freeze technique. Fig. 9 shows the power spectrum for this layer and, for comparison, a power spectrum for an approximately $150 \mu \mathrm{m}$ thick DT layer grown by the slow freeze technique. The IR generated layer has the same general modal shape as the DT layer.

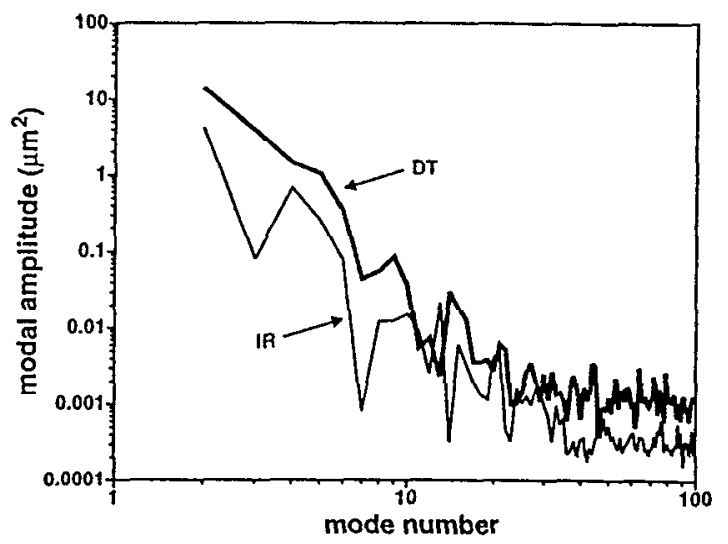

Fig. 9 Power spectrum for the layer shown in Fig. 8 along with a DT layer of comparable thickness. The noise level for these images can be seen by the flattening of the power spectrum at mode numbers above 40 .

\section{SUMMARY}

We have developed a technique to redistribute and smooth hydrogen ( $\mathrm{D}_{2}, \mathrm{HD}, \mathrm{DT}$, etc.) layers for ICF by slow solidification with simultaneous pumping of the rotation-vibration absorption bands of the solid. Relatively uniform layers can now be grown with a surface roughness comparable to that of DT. Crystals formed during a quick freeze to the solid phase are not readily smoothed by the IR heating. The solid layers slowly grown from the melt were much smoother.

\section{ACKNOWLEDGMENTS}

The authors would like to thank J. Burmann, M. Saculla, and W. Unites for their technical assistance. We would also like to thank Dr. E.R. Mapoles for providing us with a copy his FFT software modified to analyze our data. This work was performed under the auspices of the U.S. Department of Energy under contract no. W-7405Eng-48 and contract no. DE-AC03-95SF20732 by LLNL and Schafer.

\section{REFERENCES}

1. J. Lind, Phys. of Plasmas, V2, N11:3933-4024 (1995). E. M. Campbell, J. C. Browne, Science 271 130-132 (1996).
2. A. J. Martin, R. J. Simms, and R. B. Jacobs, J. Vac. Sci. Technol. A 6 (3), 1885 (1988); J. K. Hoffer and L. R. Foreman, Phys. Rev. Lett. 60, 1310 (1988); T. P. Bernat, E. R. Mapoles, and J. J. Sanchez, ICF Quarterly 1, 57 (1991).

3. G. W. Collins, E. R. Mapoles, J. Sanchez, R. Bell, W. Unites, J. Hoffer, L. Forman, and J. Simpson, "Solid Hydrogen Surfaces," ICF Quarterly 3, 81 (1993).

4. Solids Far From Equilibrium, edited by C. Godreche, (Cambridge University Press., Cambridge, 1992). In this paper we ignore effects from ${ }^{3} \mathrm{He}$ born in the solid. The effects of ${ }^{3} \mathrm{He}$ cannot be ignored in DT layers which have aged for longer than $\sim$ a day.

5. C. Y. Kuo, R. J. Kerl, N. D. Patel, and C. K. N. Patel, Phys. Rev. Lett. 53, 2575 (1984).

6. H. P. Gush, W. F. J. Hare, E. J. Allin, and J. L. Welsh, Can. J. Phys. 38, 176 (1960).

7. G.W. Collins, D.N. Bittner, E. Monsler, S. Letts, E.R. Mapoles, and T.P. Bernat, J. Vac. Sci. Technol. A, 14 (5), 2897 (1996).

8. R.B. Stephens and G. Collins, "Analysis of Integrating Sphere Performance for IR Enhanced D'T Layering", Fusion Technol., 31, 485 (1997).

9. Infragold ${ }^{1 M}$ is a electrochemically plated, diffuse, gold-metallic coating. For information contact Labsphere, North Sutton, NH 03260.

10. General Atomics Inertial Confinement Fusion Annual Report, (GA-A22816, UC-712) 5-7 (1997).

11. Raytracing was performed using the TraceProtM commercial optical modeling program. For further information contact Lambda Research Corp., Littleton, MA 01460.

12. After pyrolysis the shells were inspected and measured using a microscope which was calibrated using a $1 \mathrm{~mm}$ diam. reference sphere.

13. J. Sater, B. Kozioziemski, G.W. Collins, E.R. Mapoles, J. Pipes, "Cryogenic D-T fuel layers formed in $1 \mathrm{~mm}$ spheres by beta-layering", Fusion Technol., to be published. 